\title{
The COPD patient who couldn't stop worrying
}

Krista E. Martinez, MD, David S. Harnett, MD, and Alexis Freedberg, MD

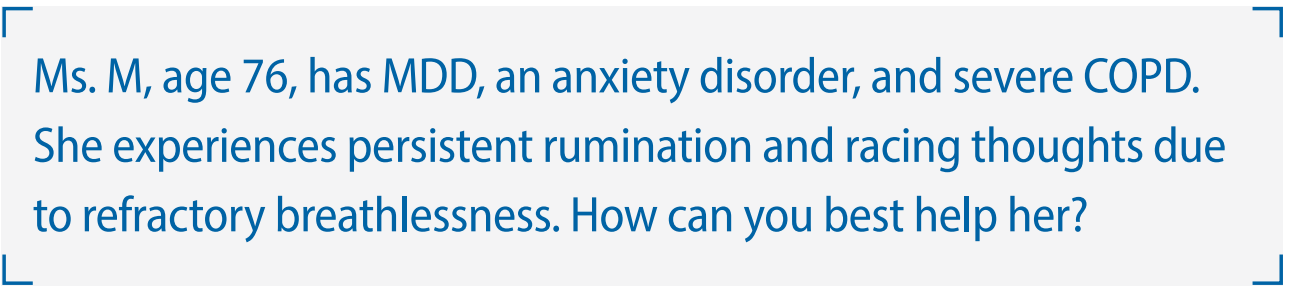

\section{CASE A passive wish to die}

Ms. $M$, age 76 , has a history of major depressive disorder, unspecified anxiety disorder, and severe chronic obstructive pulmonary disease (COPD), for which she requires supplemental oxygen. She is admitted to a psychiatric hospital after several months of increased dysphoria, rumination, anhedonia, and a passive wish to die. She also has a decreased appetite and has lost $10 \mathrm{lb}$, experiences frequent daily episodes of shortness of breath and associated racing thoughts, and has a rapid heart rate.

\section{HISTORY Past medication trials}

In addition to COPD, Ms. M's medical history includes hypertension. Past psychotropic medication trials used to treat her depression and anxiety have included aripiprazole, $5 \mathrm{mg} / \mathrm{d}$; duloxetine, $60 \mathrm{mg} / \mathrm{d}$; fluoxetine, $40 \mathrm{mg} / \mathrm{d}$; mirtazapine, $30 \mathrm{mg}$ nightly; buspirone, $10 \mathrm{mg}$ twice daily; and clonazepam, 0.5 mg twice daily. She has no history of psychotherapy, and because of her uncontrolled anxiety and depression, she has never completed a pulmonary rehabilitation program.

Her current medications include salmeterol, 50 mcg inhaled twice daily, for COPD; amlodipine, $10 \mathrm{mg} / \mathrm{d}$, for hypertension; buspirone, $10 \mathrm{mg}$ twice daily, for anxiety; and duloxetine, $60 \mathrm{mg} / \mathrm{d}$, for depression.

\section{EXAMINATION No evidence \\ of dementia}

On examination, Ms. $\mathrm{M}$ is alert and oriented to person, place, date, and situation. Overall, she has mild difficulty with attention and short-term recall, which appears to be due to poor effort; intact long-term memory; and is able to abstract appropriately. There is no evidence of dementia.

A mental status exam reveals a frail, elderly woman with fair-to-poor hygiene, cooperative behavior, slowed motor activity, slowed speech with low volume, low mood, and depressed affect with constricted range. Her thought process is linear, her thought content includes passive death wishes, and she does not have hallucinations.

Bitemporal electroconvulsive therapy (ECT), 1.0 ms pulse width at 1.5 times Ms. M's seizure threshold 3 times weekly, is initiated to treat her depression, with seizure duration averaging 45 seconds for each session. She receives a total of 8 treatments over

Dr. Martinez is a PGY-3 Psychiatry Resident, Tufts Medical Center, and Clinical Associate in Psychiatry, Tufts University School of Medicine, Boston, Massachusetts. Dr. Harnett is Clinical Professor of Psychiatry, Tufts University School of Medicine, Boston, Massachusetts. Dr. Freedberg is Clinical Assistant Professor of Psychiatry, Tufts University School of Medicine, Boston, Massachusetts.

\section{Disclosures}

The authors report no financial relationships with any companies whose products are mentioned in this article, or with manufacturers of competing products.

doi: $10.12788 / \mathrm{cp} .0067$

\section{How would you handle this case?}

Answer the challenge questions at MDedge.com/ psychiatry and see how your colleagues responded 


\section{Clinical Point}

In patients with COPD, it can be difficult to discern if a symptom is attributable to the COPD, a psychiatric diagnosis, or a combination of both

Discuss this article at www.facebook.com/ MDedgePsychiatry the course of admission. Buspirone, $10 \mathrm{mg}$ twice daily, is stopped shortly after admission, but she continues to receive duloxetine, 60 $\mathrm{mg} / \mathrm{d}$. Ms. M continues to have shortness of breath, palpitations, fearful ruminations about the future, and difficulty falling asleep.

\section{How would you treat Ms. M's refractory anxiety?}
a) lorazepam
b) exercise
c) meditation and mindfulness techniques
d) cognitive-behavioral therapy (CBT)

\section{The authors' observations}

The treatment team explores other options, such as benzodiazepines, psychotherapy modalities, and mindfulness exercises, to treat Ms. M's anxiety and comorbid COPD. Lorazepam, $0.5 \mathrm{mg}$ twice daily, was chosen to treat her acute anxiety. Due to Ms. M's need for supplemental oxygen, the treatment team attempted to mitigate the risk of using a benzodiazepine by limiting its use to the minimum effective dose. The teams also looked for alternative therapies.

Evaluation of anxiety and depression in a patient with COPD is complicated by a high degree of symptom overlap. Patients with COPD may experience anxiety symptoms such as shortness of breath, rapid heart rate, numbness/tingling, and racing thoughts, and/or depressive symptoms such as decreased energy, impaired sleep, and impaired concentration. It can therefore be difficult to discern if a symptom is attributable to the physical diagnosis, the psychiatric diagnosis, or a combination of both. Catastrophic thinking about mild physical symptoms is common in patients with COPD. This can lead to hyperventilation and hypocapnia (manifested by lightheadedness, dizziness, paresthesia, and altered consciousness), with a reciprocally escalating cascade of anxiety and somatic symptoms. ${ }^{1}$
First-line therapy for anxiety disorders with comorbid COPD is CBT and other nonpharmacologic interventions. ${ }^{2,3}$ However, access to CBT-trained psychotherapists is limited due to the cost and a shortage of qualified professionals. ${ }^{4}$ One randomized clinical trial evaluated improving accessibility to this therapy by training respiratory therapists to perform CBT sessions for COPD patients who were anxious and depressed. ${ }^{4}$ This method was as successful as using CBT-trained psychotherapists, and also reduced emergency room visits and hospitalizations in this population. ${ }^{4}$ Respiratory therapists often intuitively use CBT concepts in coaching patients and generally have the knowledge and background to be able to help patients identify symptoms caused by anxiety vs when to seek additional medical support. Furthermore, these interventions have been shown to increase quality-adjusted life years, ${ }^{4}$ improve physical performance, and reduce symptoms of anxiety and depression as reported on the Beck Anxiety Inventory and Beck Depression Inventory. ${ }^{5}$ In addition to relaxation/imagery training, cognitive restructuring, breathing retraining, and maintaining daily symptom logs, CBT for COPD may include interoceptive exposure therapy. This technique involves deliberately inducing hyperventilation, followed by desensitization exercises to uncouple any uncomfortable physical sensations with the conditioned fear response. ${ }^{1}$

Although there is little evidence that traditional pharmacologic treatments (eg, antidepressants, benzodiazepines) have a statistically significant effect on anxiety and depression in COPD, studies have found that they have some clinical benefit. $^{3}$ Risks, however, limit the utility of certain agents. Sedative-hypnotics potentially decrease respiratory drive and, particularly in older patients, antidepressants' sedating effects can increase the risk of falls ${ }^{3}$ leading to increased morbidity, hospitalization, and mortality. 


\section{TREATMENT Mindfulness techniques} and meditation

Ms. M's symptoms show no improvement with the addition of lorazepam, $0.5 \mathrm{mg}$ twice daily. A clinician teaches Ms. M mindfulness techniques, and she begins a trial of daily, individual, guided meditation using a meditation app. Respiratory therapists also instruct her on controlled breathing techniques such as pursed-lips breathing, diaphragmatic breathing, and deep breathing. They also encourage Ms. M to participate in the daily exercise group while on the unit.

\section{Which medication is effective for treating refractory breathlessness in patients with COPD?}
a) a once-daily opioid
b) levodopa
c) naloxone
d) no medication has been found to be helpful

\section{The authors' observations}

Research indicates that low doses of opioids are safe and effective for refractory breathlessness in patients with severe COPD (those with an arterial partial pressure of oxygen $\leq 55 \mathrm{~mm} \mathrm{Hg}$ or arterial oxygen saturation $\leq 88 \%$ ). ${ }^{6,7}$ Patients at this advanced stage of illness have likely been started on oxygen therapy to improve survival but may need additional palliative measures to alleviate the discomfort associated with the severe breathlessness experienced at this stage. ${ }^{8}$ In such situations, low-dose opioids, such as immediate-release morphine, $1 \mathrm{mg} / \mathrm{d}$ given as $0.5 \mathrm{mg}$ twice daily, can be started. ${ }^{8}$ The dose can be increased by $0.5 \mathrm{mg}$ until a therapeutic dose is achieved. ${ }^{8}$ Alternately, 24-hour extended-release morphine, $20 \mathrm{mg} / \mathrm{d}$, or an equivalent dose of oxycodone or hydromorphone, can be prescribed. ${ }^{8}$ The proposed therapeutic mechanisms of low-dose opioids for these patients include decreasing one's sense of effort, altering central perception, altering activity

\section{Table}

\section{Ways to safeguard against opioid abuse}

Avoid opioid use in patients who have also been prescribed a benzodiazepine or other sedative-hypnotic medications

Limit the amount of the opioid prescribed

Provide psychoeducation for the patient and family members

Assess for signs that the medication is being diverted

of peripheral opioid receptors located in the lung, and decreasing anxiety. ${ }^{8}$

The current opioid crisis prompts additional caution in prescribing, especially when considering using short-acting, immediate-release opioids such as morphine, which have a greater potential for abuse and dependence. The Table lists safeguards that should be implemented when prescribing opioids.

Many patients with COPD in the endof-life phase and in severe pain or discomfort due to the advanced stages of their illness receive opioids as part of palliative care. Patients with COPD whose medical care is predominantly palliative may benefit greatly from being prescribed opioids. Most patients with COPD who find relief from low-dose opioids usually have 6 to 12 months to live, and low-dose opioids may help them obtain the best possible quality of life.

Choosing opioids as a treatment involves the risk of physiologic dependence and opioid use disorder. For Ms. M, the potential benefits were thought to outweigh such risks.

\section{OUTCOME Breathlessness improves, anxiety decreases}

Ms. M's lorazepam is discontinued, and immediate-release morphine is prescribed at a low dose of $1 \mathrm{mg} / \mathrm{d}$ on an as-needed basis

\section{Clinical Point}

First-line therapy for anxiety disorders with comorbid COPD is CBT and other nonpharmacologic interventions 


\section{Clinical Point}

\section{Research indicates that low doses of opioids are safe and effective for refractory breathlessness in patients with severe COPD}

\section{Related Resources}

- Alexopoulos G, Kiosses D, Sirey J, et al. Untangling therapeutic ingredients of a personalized intervention for patients with depression and severe COPD. Am J Geriatr Psychiatry. 2014;22(11):1316-1324.

- Jackson D, Banerjee S, Sirey J, et al. Two interventions for patients with major depression and severe chronic obstructive pulmonary disease: impact on quality of life. Am J Geriatr Psychiatry. 2018;27(5):502-511.

\section{Drug Brand Names}

Amlodipine • Norvasc

Aripiprazole • Abilify

Buspirone • Buspar

Clonazepam $\cdot$ Klonopin

Duloxetine - Cymbalta

Fluoxetine - Prozac

Hydromorphone • Dilaudid

Levodopa $\cdot$ Sinemet
Lorazepam $\cdot$ Ativan
Mirtazapine $\cdot$ Remeron
Morphine $\cdot$ MS Contin
Naloxone $\cdot$ Narcan
Oxycodone $\cdot$ Oxycontin
Salmeterol $\cdot$ Serevent Diskus

for anxiety with good effect. Ms. M's breathlessness improves, leading to an overall decrease in anxiety. She does not experience sedation, confusion, or adverse respiratory effects.

Ms. M's anxiety and depression improve over the course of the hospitalization with this regimen. On hospital Day 25, she is discharged with a plan to continue duloxetine, $60 \mathrm{mg} / \mathrm{d}$, ECT twice weekly, and low-dose morphine, $1 \mathrm{mg} / \mathrm{d}$, as needed for anxiety. She is referred for pulmonary rehabilitation and CBT to maintain remission.

\section{Which of the following has the greatest impact on reducing morbidity and mortality in patients with COPD?}
a) supplemental oxygen
b) pulmonary rehabilitation
c) access to an academic medical center
d) increased frequency of hospitalizations for COPD exacerbation

\section{The authors' observations}

Ms. M's case highlights several challenges associated with treating psychiatric illness in a patient with a chronic medical illness. The relationship between COPD, anxiety, and depression is complex, and is associated with reduced quality of life, increasing severity of pulmonary disease, increased dyspnea, a sense of loss and inability to cope, and decreased self-efficacy and adherence to treatment. ${ }^{9-11}$ Adding supplemental oxygen has been shown to improve longevity, and may help boost mood and lessen anxiety in some patients, whereas others find its restrictions burdensome and demoralizing. ${ }^{8}$ Pulmonary rehabilitation programs are a key component of both secondary prevention and treatment in improving overall health outcomes in patients with COPD, ${ }^{12}$ and can be started at any stage of the illness. However, patients with comorbid anxiety and depression are often the least likely to engage in these programs. ${ }^{4,13}$ One program that offered management of depression and COPD care with a focus on misconceptions about treatment and obstacles to treatment engagement reduced depressive symptoms and dyspnea-related disability, which led to greater remission of depression. ${ }^{14,15}$ Exercise training in such programs also helps manage avoidance of physical activity due to fear of dyspnea by allowing patients to experience feared symptoms in the presence of medical professionals, similar to the CBT interoceptive exposure technique described earlier. ${ }^{1}$ Several traditional treatment modalities used to target Ms. M's anxiety disorder were unsuccessful. Low-dose, as-needed opioids are a safe and

\section{Bottom Line}

When traditional antidepressant and anxiolytic therapies have not sufficiently helped, consider low-dose, once-daily opioids to address refractory breathlessness in a patient with COPD with comorbid anxiety and depression. This treatment can lead patients to participate in rehabilitation therapies and improve their quality of life. 
useful pharmacologic option worth considering for patients with refractory anxiety and depression and comorbid COPD.

\section{References}

1. Harnett D. The difficult-to-treat psychiatric patient with comorbid medical illness. In: Dewan M, Pies R, eds. The difficult-to-treat psychiatric patient. Washington, DC: American Psychiatric Association Publishing; 2001: 325-357.

2. Panagioti M, Scott C, Blakemore A, et al. Overview of the prevalence, impact, and management of depression and anxiety in chronic obstructive pulmonary disease. Int J Chron Obstruct Pulmon Dis. 2014;9:1289-1306.

3. Cafarella P, Effing T, Usmani ZA, et al. Treatments for anxiety and depression in patients with chronic obstructive pulmonary disease: a literature review. Respirology. 2012; 17(4):627-638

4. Heslop-Marshall K, Baker C, Carrick-Sen D, et al Randomised controlled trial of cognitive behavioural therapy in COPD. ERJ Open Res. 2018;4:00094-2018. doi: 10.1183/23120541.00094-2018.

5. de Godoy DV, de Godoy RF. A randomized controlled trial of the effect of psychotherapy on anxiety and depression in chronic obstructive pulmonary disease. Arch Phys Med Rehabil. 2003;84(8):1154-1157.

6. Abernethy A, Currow D, Frith P, et al. Randomised, double blind, placebo controlled crossover trial of sustained release morphine for the management of refractory dyspnoea. BMJ. 2003;327(7414):523-528.

7. Janowiak P, Krajnik M, Podolec Z, et al. Dosimetrically administered nebulized morphine for breathlessness in very severe chronic obstructive pulmonary disease: a randomized, controlled trial. BMC Pulm Med. 2017; 17:186.

8. Rocker G, Horton R, Currow D, et al. Palliation of dyspnoea in advanced COPD: revisiting a role for opioids. Thorax. 2009;64(10):910-915.

9. Pooler A, Beech R. Examining the relationship between anxiety and depression and exacerbations of COPD which result in hospital admission: a systematic review. Int J Chron Obstruct Pulmon Dis. 2014;9:315-330.

10. Carmen Valenza M, Valenza-Peña G, Torres-Sánchez I, et al. Effectiveness of controlled breathing techniques on anxiety and depression in hospitalized patients with COPD: a randomized clinical trial. Respir Care. 2014;59(2):209-215.

11. Pollok J, van Agteren J, Esterman A, et al. Psychological therapies for the treatment of depression in chronic obstructive pulmonary disease. Cochrane Database Syst Rev. 2019;3:CD012347. doi: 10.1002/ 14651858.CD012347.pub2.

12. Roberts N, Kidd L, Kirkwood K, et al. A systematic review of the content and delivery of education in pulmonary rehabilitation programmes. Respiratory Medicine. 2018;145: 161-181.

13. Pumar M, Gray C, Walsh J, et al. Anxiety and depressionimportant psychological comorbidities of COPD. J Thorac Dis. 2014;6(11):1615-1631

14. Alexopoulos G, Kiosses D, Sirey J, et al. Untangling therapeutic ingredients of a personalized intervention for patients with depression and severe COPD. Am J Geriatr Psychiatry. 2014;22(11):1316-1324.

15. Jackson D, Banerjee S, Sirey J, et al. Two interventions for patients with major depression and severe chronic obstructive pulmonary disease: impact on quality of life. Am J Geriatr Psychiatry. 2018;27(5):502-511.

\section{Clinical Point}

Patients with comorbid COPD and anxiety are the least likely to engage in pulmonary rehabilitation programs

\section{AD INDEX}

Eisai Inc.

Dayvigo

PP 11-14

Janssen

Spravato

C3-C4, PP 46-52

Otsuka America Pharmaceutical,Inc

Abilify Maintena

.PP 1-7

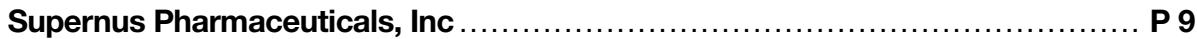

United States Army 